\title{
Some Properties of Graph of a Finite Group
}

\author{
R.A.Muneshwar ${ }^{1}$, S.S.Agrawal' ${ }^{2}$, S.G.Jakkewad ${ }^{3}$, \\ ${ }^{I}$ Department of Mathematics, NES Science College, SRTMU Nanded, India - 431602 \\ ${ }^{2}$ Department of Mathematics, NES Science College, SRTMU Nanded, India - 431602 \\ ${ }^{3}$ Department of Mathematics, NES Science College, SRTMU Nanded, India - 431602
}

\begin{abstract}
In this paper we introduced a new concept of graph of any finite group and we obtained graphs of some finite groups. Moreover some results on this concept are proved.

Keywords: Group, Abelian group, Cyclic group, Graph, Degree of a vertex, Degree of a graph.
\end{abstract}

\section{Introduction:}

The origin of graph theory started with the problem of Koinsberg bridge, in 1735. This problem lead to the concept of Eulerian graph. Euler studied the problem of Koinsberg bridge and constructed a structure to solve the problem called Eulerian graph. In 1840, A.F Mobius gave the idea of complete graph and bipartite graph and Kuratowski proved that they are planar by means of recreational problems. The concept of tree was implemented by Gustav Kirchhoff in 1845, and he employed graph theoretical ideas in the calculation of currents in electrical networks or circuits. In 1852, Thomas Gutherie found the famous four color problem. Then in 1856, Thomas. P. Kirkman and William R.Hamilton studied cycles on polyhydra and invented the concept called Hamiltonian graph by studying trips that visited certain sites exactly once. In 1913, H.Dudeney mentioned a puzzle problem. Eventhough the four color problem was invented it was solved only after a century by Kenneth Appel and Wolfgang Haken. This time is considered as the birth of Graph Theory [1].

Caley studied particular analytical forms from differential calculus to study the trees. This had many implications in theoretical chemistry. This lead to the invention of enumerative graph theory. Any how the term "Graph" was introduced by Sylvester in 1878 where he drew an analogy between "Quantic invariants" and covariants of algebra and molecular diagrams. In 1941, Ramsey worked on colorations which lead to the identification of another branch of graph theory called extremel graph theory. In 1969, the four color problem was solved using computers by Heinrich. The study of asymptotic graph connectivity gave rise to random graph theory.[1]

Muneshwar R. A. and Bondar K.L. in [2] introduced the concept of graph of a finite group. Some properties of graph of finite group are proved. In this paper some more properties with example are discussed.

\section{Preliminary Notes:}

Following definitions are comes from references [3], [4], [5], [6], [7], [8] [9].

Definition 2.1 (Group): A nonempty set $G$ with a binary operation . is called as a group if the following axioms hold:

(i) $a(b c)=(a b) c$ for all $a, b, c \in G$

(ii) There exists $e$ in $\mathrm{G}$ such that $e a=a e=a ; \forall a \in G$

(iii) For every $a \in G$ there exists $a^{\prime} \in G$ such that $a^{\prime} a=a a^{\prime}=e$.

Definition 2.2 (Abelian group): A group $G$ in which all elements satisfies commutative law is called as an abelian group.

Definition 2.3 (Cyclic group): A group $G$ is said to be cyclic if $G=[a]=\left\{x=a^{n} \mid n \in Z\right\}$, for some $a \in G$. The most important examples of cyclic groups are the additive group $Z$ of integers and the additive groups $Z /(n)$ of integers modulo $n$. In fact, these are the only cyclic groups up to isomorphism.

Definition 2.4 (Subgroup): Let $(G$, .) be a group and $H$ be a subset of $G$. Then $H$ is called a subgroup of $G$, if $H$ is a group relative to the binary operation in $G$ and it is denoted by $H \leq G$.

Definition 2.5 (Center of a group): The center of a group $G$, written as $Z(G)$, is the set of those elements in $G$ that commute with every clement in $G$. That is $Z(G)=(a \in G \mid a x=x a \forall x \in G\}$.

Definition 2.6 (Centralizer of an element): Let $g \in G$ be any element of group $G$ then centralizer of an element is written as $C(g)$, is the set of those elements in $G$ that commute with element $g$. i.e. $C(g)=\{a \in G \mid a g=g a\}$.

Definition 2.7 (Centralizer of a subgroup): Let $H$ be any subgroup of $G$ then centralizer of a subgroup is written as $C(H)$, is the set of those elements in $G$ that commute with all elements of subgroup $H$.

i.e. $C(H)=\{a \in G \mid a h=h a, \forall h \in H\}$. 
Definition 2.8 (Order of an element): Let $G$ be a group, and $a \in G$. If there exists a least positive integer $m$ such that $a^{m}=e$, then such a positive integer $m$ is called as order of $a$ and it is written as $o(a)$. If no such positive integer exists, then $a$ is said to be of infinite order.

Definition 2.9 (Order of a group): Number of elements in a group $G$ is called as order of a group and it is denoted by $o(G)$ or $|G|$. If order of a group is finite then group is said to be finite group and if order of a group is infinite then group is said to be infinite group.

Definition 2.10 (Graph): Graph be an ordered pair $G=(V, E)$, where $V$ be a set of vertices of graph and $E$ be a set of edges of graph. The vertices $g_{i}, g_{j}$ associated with edge $e_{\mathrm{k}}$ are called as end vertices of $e_{k}$.

Definition 2.11 (Degree of a vertex): Number of edges incident on vertex $g_{k}$ with loop counted twice is called as degree of a vertex $g_{k}$, and it is denoted by $d\left(g_{k}\right)$.

Definition 2.12 (Degree of a graph): Sum of degree of all vertices of a graph $\mathrm{G}$ is called as degree of a graph $\mathrm{G}$ and it is denoted by $d(G)$.

Definition 2.13 (Graph of a finite group): Let $\mathrm{G}$ is a finite group of order $\mathrm{n}$. Then graph of $\mathrm{G}$ is denoted by $\mathrm{R}(\mathrm{G})$ and is defined as $R(G)=(R(V), R(E))$, where

1. $R(V)=$ set of vertices of graph of $G=G$. and

2. $R(E)=$ set of edges of graph of $G$

$=\left\{r_{i j} \mid r_{i j}\right.$ is an edge between $g_{i}$ and $g_{j}$ if and only if $g_{i}$ and $g_{j}$ are commute in group $\}$

Example 2.1: If $\mathrm{G}=Z_{4}=\{0,1,2,3\}$ be an abelian group of order 4. Let $R(G)=\{R(V), R(E)\}$ be graph of a group $G$, where $R(V)=G=Z_{4}=\{0,1,2,3\}$ and

$R(E)=\left\{r_{i j}=\left(g_{i,} g_{j}\right) \mid g_{i}\right.$ and $g_{j}$ are commutes in a group $\}$

i.e. $R(E)=\left\{r_{i j}=\left(g_{i}, g_{j}\right) \mid g_{i} g_{j}=g_{j} g_{i} \forall i, j\right\}$

Hence $R(E)=\{(0,0),(1,1),(2,2),(3,3),(0,1),(0,2),(0,3),(1,2),(1,3),(2,3)\}$

Thus the graph of $G$ is as follows.

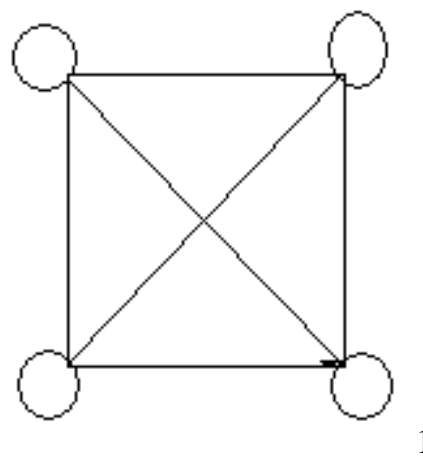

Fig. 1

Example 2.2: If $G=D_{3}=\left\{e, a, a^{2}, r_{1}, r_{2}, r_{3}\right\}$ be a non abelian group of order 6

Let $R(G)=\{R(V), R(E)\}$ be graph of a group $G$, where $R(G)=G=D_{3}=\left\{e, a, a^{2}, r_{1}, r_{2}, r_{3}\right\}$ and $R(E)=\left\{r_{i j}=\left(g_{i}, g_{j}\right) \mid g_{i}\right.$ and $g_{j}$ are commute in a group $\left.\mathrm{G}\right\}$

Hence, $R(E)=\left\{(e, e),(e, a),\left(e, a^{2}\right),\left(e, r_{1}\right),\left(e, r_{2}\right),\left(e, r_{3}\right),(a, a),\left(a, a^{2}\right),\left(r_{1}, r_{1}\right),\left(r_{2}, r_{2}\right),\left(r_{3}, r_{3}\right)\right\}$

Thus the graph representation of $G=D_{3}$ is as follow 


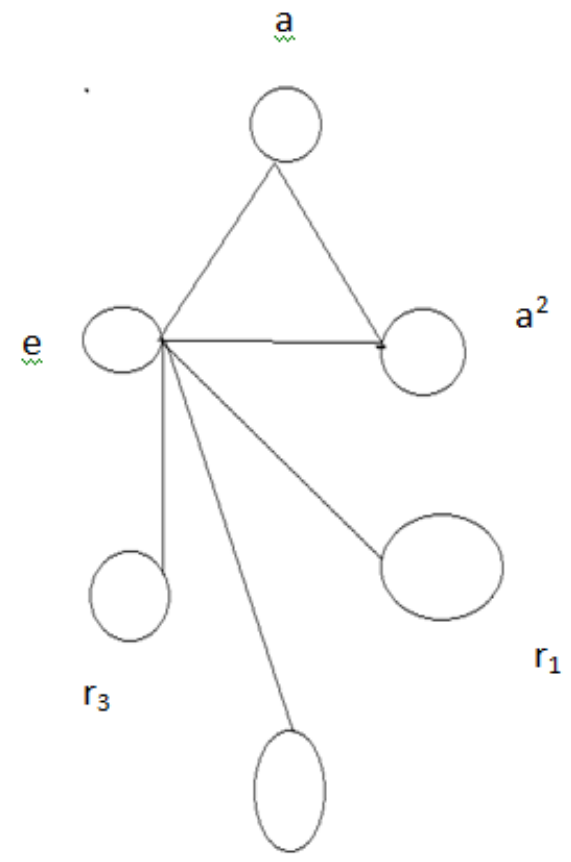

$\mathrm{r}_{2}$

Following results have been proved in [2].

Fig. 2

Theorem 2.1: If $G$ be any group of order $n$ then $o\left(C\left(g_{i}\right)\right)=d\left(g_{i}\right)-1$.

Theorem 2.2. If $G$ be any group of order $n$ with identity elements $e$ then $d(e)=o(G)+1$.

Theorem 2.3: If $G$ be any abelian group of order $n$ then $d\left(g_{i}\right)=o(G)+1 \quad ; \forall g_{i} \in G$

Theorem 2.4: If $G$ be any abelian group of order $n$ then $d(G)=o(G)(o(G)+1)$.

\section{3. Main Result:}

Theorem 3.1: If $G$ is a group of order $n$ such that $d(G)=o(G)[o(\mathrm{G})+1]$ then prove that, $G$ is abelian group.

Proof: Let $G$ be a group of order $n$ such that,

$$
d(G)=o(G)[o(\mathrm{G})+1]
$$

i.e. $d(G)=[o(\mathrm{G})+1]+[o(\mathrm{G})+1]+\ldots+[o(\mathrm{G})+1](n$ times $)$

i.e. $d(G)=[o(\mathrm{G})+o(\mathrm{G})+\ldots+o(\mathrm{G})](n$ times $)+\mathrm{o}(G)$-------(1)

But $d(G)=\sum_{k=1}^{n} o\left(C\left(g_{k}\right)\right)+o(G)$.

i.e. $d(G)=\left[o\left(C\left(g_{1}\right)\right)+o\left(C\left(g_{2}\right)\right)+\ldots+o\left(C\left(g_{n}\right)\right)\right]+\mathrm{o}(G)$------- (2)

From equations (1) and (2), we have

$[o(\mathrm{G})+o(\mathrm{G})+\ldots+o(\mathrm{G})](n$ times $)+\mathrm{o}(G)=\left[o\left(C\left(g_{1}\right)\right)+o\left(C\left(g_{2}\right)\right)+\ldots+\mathrm{o}\left(C\left(g_{n}\right)\right)\right](n$ times $)+\mathrm{o}(G)$

Hence $o\left(C\left(g_{k}\right)\right)=o(\mathrm{G}) \quad ; \forall g_{k} \in G$

i.e. $C\left(g_{k}\right)=\mathrm{G} ; \forall g_{k} \in G$

i.e. $\mathrm{g}_{\mathrm{k}}$ is adjacent with all vertices of graph of $\mathrm{G} ; \forall g_{k} \in G$

i.e. $g_{k}$ is commutes with all elements of group $\mathrm{G} ; \forall g_{k} \in G$

i.e. every element of group is commutes with all other elements of group $\mathrm{G}$

Thus group $\mathrm{G}$ is abelian group.

From Theorem 2.4 and Theorem 3.1, now we can define an abelian group is as follows.

(Definition) Abelian group: If $G$ is a group of order $n$ then a group $G$ is called as an abelian group if and only if $\mathrm{d}(\mathrm{G})=\mathrm{o}(\mathrm{G})[\mathrm{o}(\mathrm{G})+1]$.

Theorem 3.2: If $G$ is a group of order $n$ such that $d(G)<o(G)[o(\mathrm{G})+1]$ then prove that $G$ is non abelian group.

Proof : Let $G$ be any group of order $n$ such that

$$
d(G)<o(G)[o(\mathrm{G})+1]
$$

i.e. $d(G)=[o(\mathrm{G})+1]+[o(\mathrm{G})+1]+\ldots+[o(\mathrm{G})+1](n$ times $)$

i.e. $d(G)=[o(\mathrm{G})+o(\mathrm{G})+\ldots+o(\mathrm{G})](n$ times $)+\mathrm{o}(G)------(1)$

But $d(G)=\sum_{k=1}^{n} o\left(C\left(g_{k}\right)\right)+o(G)$

i.e. $d(G)=\left[o\left(C\left(g_{1}\right)\right)+o\left(C\left(g_{2}\right)\right)+\ldots+o\left(C\left(g_{n}\right)\right)\right]+\mathrm{o}(G)$ 
From equations (1) and (2), we have

$[o(\mathrm{G})+o(\mathrm{G})+\ldots+o(\mathrm{G})](n$ times $)+\mathrm{o}(G)>\left[o\left(C\left(g_{1}\right)\right)+o\left(C\left(g_{2}\right)\right)+\ldots+o\left(C\left(g_{n}\right)\right)\right](n$ times $)+\mathrm{o}(G)$

Hence $o\left(C\left(g_{k}\right)\right)<o(\mathrm{G})$; for some $\mathrm{k}$

i.e. $C\left(g_{k}\right) \neq \mathrm{G}$; for some $\mathrm{k}$

i.e. $\mathrm{G} \neq\left\{g_{i} \in G \mid g_{i}\right.$ is not adjacent with $\left.g_{k}\right\}$; for some $\mathrm{k}$

i.e. $\mathrm{G} \neq\left\{g_{i} \in G \mid g_{i} g_{k}=\mathrm{g}_{\mathrm{k}} \mathrm{g}_{\mathrm{i}}\right\}$; for some $\mathrm{k}$

i.e. $\exists$ at least one vertex $g_{i}$ such that $g_{i} g_{k} \neq g_{k} g_{i}$

Thus group $\mathrm{G}$ is non abelian.

Theorem 3.3: If $G$ is a group of order $n$ and $g_{k}$ is any element of group $\mathrm{G}$ such that $g_{k}$ is not commutes with some $g_{i}$ then $d(G)<o(G)[o(\mathrm{G})+1]$ and group $\mathrm{G}$ is non abelian.

Proof: Let $G$ be a group of order $n$ and $g_{k}$ is any element of group $\mathrm{G}$ such that $g_{k}$ is not commutes with some $g_{i}$.

i.e. $g_{i} g_{k} \neq g_{k} g_{i}$; for some i

i.e. There is no edge between $g_{i}$ and $g_{k}$.

i.e. $C\left(g_{k}\right) \neq \mathrm{G}$

i.e. o $\left(C\left(g_{k}\right)\right) \neq \mathrm{o}(G) \quad$; for some $k$

i.e. o $\left(C\left(g_{k}\right)\right)+1 \neq \mathrm{o}(G)+1 \quad$; for some $k$

i.e. $d\left(g_{k}\right) \neq \mathrm{o}(G)+1 \quad$; for some $k$

i.e. $d\left(g_{k}\right)<\mathrm{o}(G)+1 \quad$; for some $k$

i.e. $\sum_{k=1}^{n} d\left(g_{k}\right)<\sum_{k=1}^{n}(\mathrm{o}(G)+1)$

i.e. $d(G)<o(G)[o(\mathrm{G})+1]$

Thus by Theorem 3.2, a group $\mathrm{G}$ is non abelian.

From Theorem 3.2 and Theorem 3.3, now we can define non abelian group is as follows.

(Definition) Non Abelian group: If $G$ is a group of order $n$ then $G$ is non abelian group if and only if

$$
d(G)<o(G)[o(\mathrm{G})+1] .
$$

3.4 Corollary: If $G$ is a group of order $n$ then $d(G)=o(G)[o(\mathrm{G})+1]$ if and only if $G$ is abelian group.

3.5 Corollary: If $G$ is a group of order $n$ then $d(G)=o(G)[o(\mathrm{G})+1]$ if and only if $G$ is cyclic group.

3.6 Corollary: If $G$ is a group of order $n$ then $d(G)<o(G)[o(\mathrm{G})+1]$ if and only if $G$ is non abelian group.

\section{Conclusion:}

An attempt has been made to show that graphs can be used to represent almost any problem involving discrete arrangements of objects, where concern is not with the internal properties of these objects but with the relationships among them. We try to made relationship between graph theory and group theory. In this paper we define abelian and non abelian group in the form of graph of a group. Moreover we try to study the various properties of group by using corresponding graph of group.

\section{References:}

[1] S.G. Shrinivas et. al. / International Journal of Engineering Science and Technology, Vol. 2(9), 2010, 4610-4621

[2] R. A. Muneshwar, K.L. Bondar, On Graph of a Finite Group, IOSR Journal of Mathematics,. Volume 10, Issue 5 Ver. VI (SepOct. 2014), PP 05-09.

[3] Joseph A. Gillian, Contemporary Abstract Algebra (Fourth Edition, Narosa Publishing House Pvt. Ltd. 1999)

[4] I. S. Luther and I. B. S. Passi, Algebra-Vol. 1: Groups ( Narosa Publishing House Pvt. Ltd., New Delhi, 1996. )

[5] Jean H. Gallier. Discrete Mathematics.(Universitext. Springer Verlag, first edition, 2011.)

[6] Reinhard Diestel, Graph Theory ( Springer International Publication, Second Edition, 2011)

[7] Douglas B. West, Introduction to graph theory (Eastern Economy Edition, PHL Learning Private Limited, Second Edition, 2011)

[8] Narsingh Deo, Graph Theory with applications to engineering and computer science (Eastern Economy Edition, Prentice - Hall Of India Private Limited, 2005)

[9] Pratima Panigrahi, S.B. Rao, Graph Theory Research Directions (Narosa Publishing House Pvt. Ltd. 2011). 